Melita Jesusa Suga Tuaño Uy, MD

Norberto V. Martinez, MD

Department of Otorhinolaryngology

- Head and Neck Surgery

University of Santo Tomas Hospital

España, Manila
Correspondence: Melita Jesusa Suga Tuaño Uy, MD Department of Otorhinolaryngology

-Head and Neck Surgery

University of Santo Tomas Hospital

España, Manila 1001

Phone : (632) 731-3001 local 2411

Philippines

E-mail address: suga_7200@yahoo.com

Reprints will not be available from the author.

No funding support was received for this study. The authors signed a disclosure that they have no proprietary or financial interest with any organization that may have a direct interest in the subject matter of this manuscript, or in any product used or cited in this study.

Presented at the Instrument Design Contest (2nd place) Philippine Society of Otolaryngology Head and Neck Surgery 50th Annual Convention, EDSA Shangri-la Plaza Hotel, Mandaluyong City, December 2, 2006.

\section{Model Myringotomy Practice Set: A do-it-yourself and inexpensive alternative}

\begin{abstract}
Objective: To develop a simple, portable, inexpensive model for otolaryngology trainees to practice on and develop skills required for myringotomy and tympanostomy tube insertion.

Materials and Methods: Recycled plastic egg crate, a 3-cc plastic syringe, micropore ${ }^{\mathrm{TM}}$ tape and modeling clay were used to create a model to practice myringotomy and tympanostomy tube insertion utilizing tubes fashioned from a recycled 18 guage intravenous catheter.
\end{abstract}

Result: The model myringotomy practice set is an inexpensive, simple do-it-yourself device made of locally available, mostly recycled materials.

Keywords: myringotomy practice set, myringotomy, middle ear ventilation, tympanostomy, tympanostomy tube insertion, instrumentation

MYRINGOTOMY with or without tympanostomy tube insertion is a common ambulatory procedure performed by otorhinolaryngologists. It is usually indicated for otitis media with effusion, and other specific manifestations of eustachian tube dysfunction and middle ear pathology.

Although the procedure is relatively fast, safe and easy, it still requires appropriate microsurgical skills. Unlike temporal bone dissection for learning mastoidectomy, training for myringotomy is difficult if not impossible to do in locally-available cadavers due to hardened and desiccated tissues. Models for teaching myringotomy have been developed but are usually expensive and not readily available especially in our local setting. Otorhinolaryngology residents are usually introduced to myringotomy by observation followed by practice on actual patients, with all the attendant risks and complications associated with the learning curve. We aimed to develop a simple, portable, inexpensive model for trainees to practice with and develop the skills required for myringotomy and tympanostomy tube insertion without compromising patient safety. The 


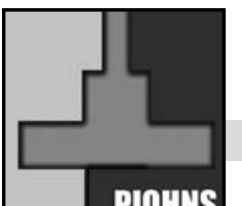

SURGICAL INNOVATIONS AND INSTRUMENTATION

Philippine Journal Of Otolaryngology-Head And Neck Surgery

VOL. 23 No. 1 JANUARY -JUNE 2008

PJOHLS

Table 1. Cost of production of the model myringotomy practice set

\begin{tabular}{l|ll} 
Grade of Impairment & \multicolumn{2}{|l}{$\begin{array}{l}\text { Corresponding audiometric ISO } \\
\text { value (Average of 500, 1000 } \\
\text { and 2000 Hz) }\end{array}$} \\
\hline Plastic egg crate & Recycled & \\
\hline 3-cc Syringe & Recycled & (PhP 5.00 if bought) \\
\hline Micropore tape & Used & (PhP 40.00 if bought) \\
\hline Modeling Clay & Recycled & (PhP 20.00 if bought) \\
\hline Intravenous Catheter & Recycled & (PhP 36.50 if bought) \\
\hline Rubber bands and paper & Recycled & \\
\hline Total & \multicolumn{2}{|c}{ (PhP 101.50 if bought) } \\
\hline
\end{tabular}

materials for this model are readily available and are inexpensive.

\section{MATERIALS AND METHODS}

\section{Materials}

1. Egg crates, plastic

2. Modeling clay

3. 3-cc plastic syringe (Terumo, Japan) for the External Auditory Canal

4. Micropore $^{\mathrm{TM}}$ Surgical Tape $\left(3 \mathrm{M}^{\mathrm{TM}}\right.$, St. Paul, MN, U.S.A.) or paraffin, cellophane or latex gloves for the Tympanic Membrane

5 . Rubber bands (to secure the tympanic membrane)

6. Intravenous Catheter 18 Gauge (Introcan-W, B.Braun Laboratories, Brazil) for making tympanostomy tubes, or recycled commercially available Tympanostomy tubes

\section{Procedure}

The syringe was cut into pieces approximately $2.5 \mathrm{~cm}$ long, and one end of each piece was beveled at around 40 degrees as shown in Figure 1. This beveled syringe would correspond to the external auditory canal.

The beveled end of each tube was wrapped with micropore ${ }^{T M}$ tape to simulate a tympanic membrane (Figure 2). Alternatively, paraffin, cellophane or latex gloves could be used and secured with a rubber band. The rest of the tube could also be wrapped with paper or aluminum foil to darken the simulated External Auditory Canal.

A moderate amount of molding clay was placed in each egg crate (Figure 3) and a depression was made to approximate the middle ear cavity, with the orifice the size of the tube. Water could be placed in this depression to simulate middle ear fluid.

The beveled end of each tube was then sealed in the clay, orienting each in a manner similar to the anatomical orientation of a patient positioned in sitting or supine position for a myringotomy (Figure 4). Tympanostomy tubes could be made out of gauge 18 IV cannulae cut into 2-mm long tubes with the ends heated to create flanges as previously described1. The model was ready to use for practice myringotomy and tympanostomy tube insertion. Suctioning of the fluid could also be practiced (Figures 5 to 9).

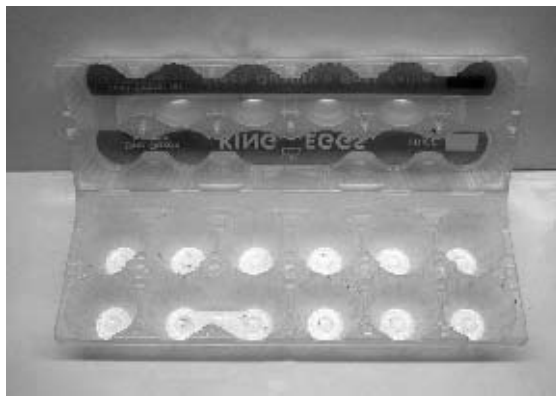

Material 1. Egg crates, plastic

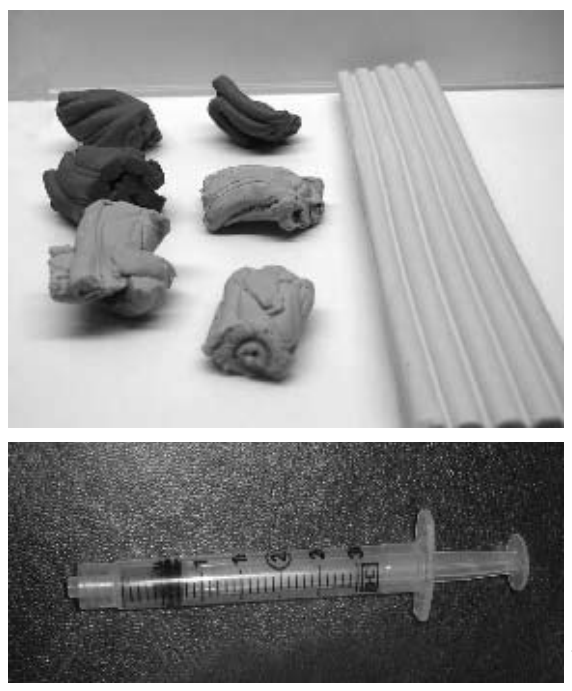

Material 2. Modeling clay

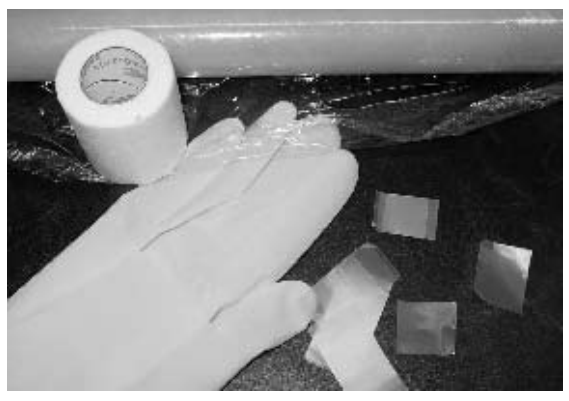

Material 4. Micropore $^{\mathrm{TM}}$ Surgical Tape $\left(3 \mathrm{M}^{\mathrm{T}}\right.$, St. Paul, MN, U.S.A.) or paraffin, cellophane or latex gloves for the Tympanic Membrane

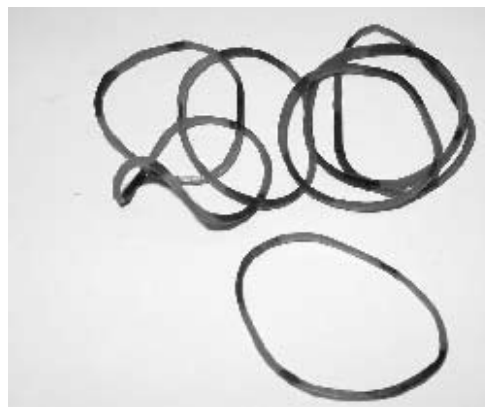

Material 5. Rubber bands (to secure the tympanic membrane)

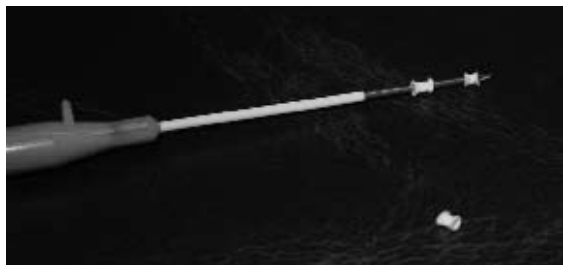

Material 6. Intravenous Catheter 18 Gauge (Introcan-W, B.Braun Laboratories, Brazil) for making tympanostomy tubes, or recycled commercially available Tympanostomy tubes 
SURGICAL INNOVATIONS AND INSTRUMENTATION

Figure 1. beveled syringe

Figure 2. beveled syringe wrapped with micropore

Figure 3. egg crate with modeling clay

Figure 4. tube in clay

Figure 5. tube in crate
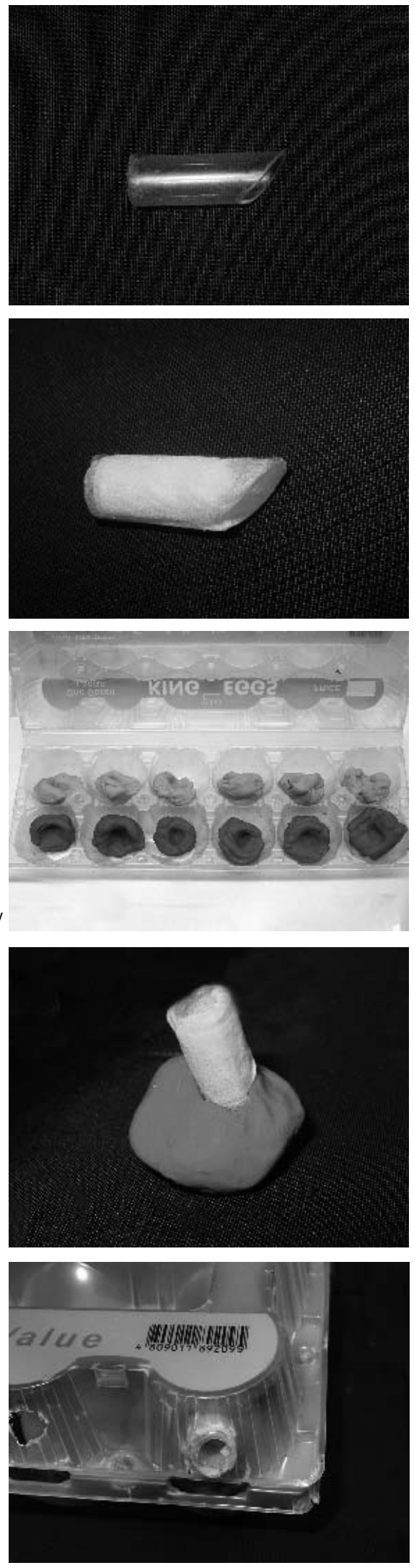
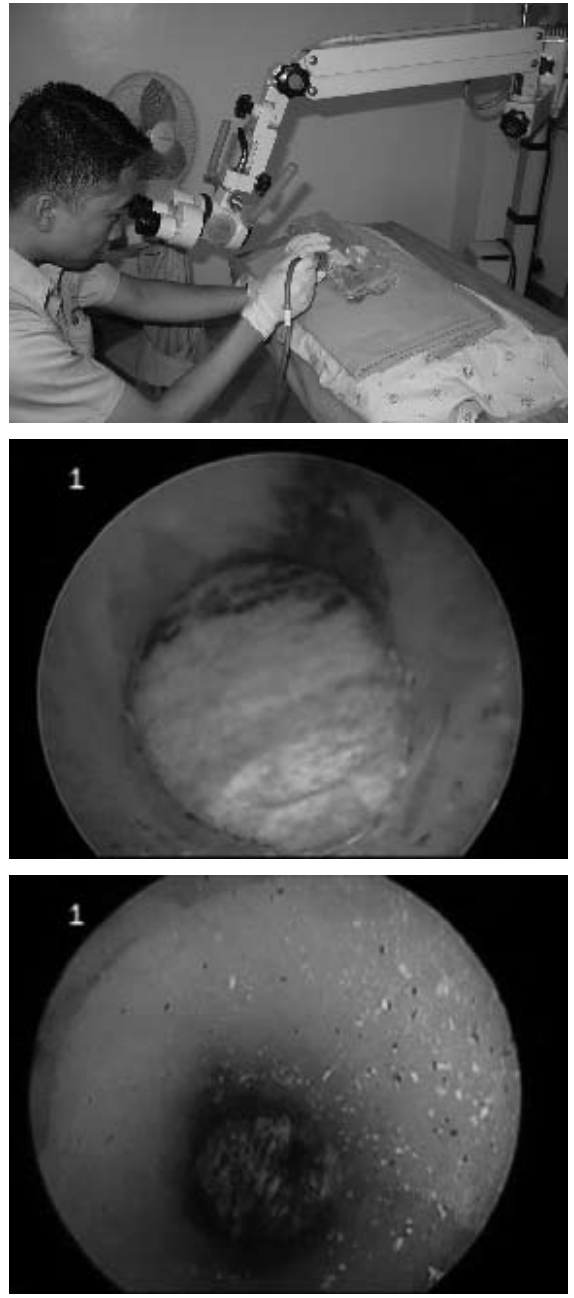

Figure 6B. Brown colored fluid behind model TM
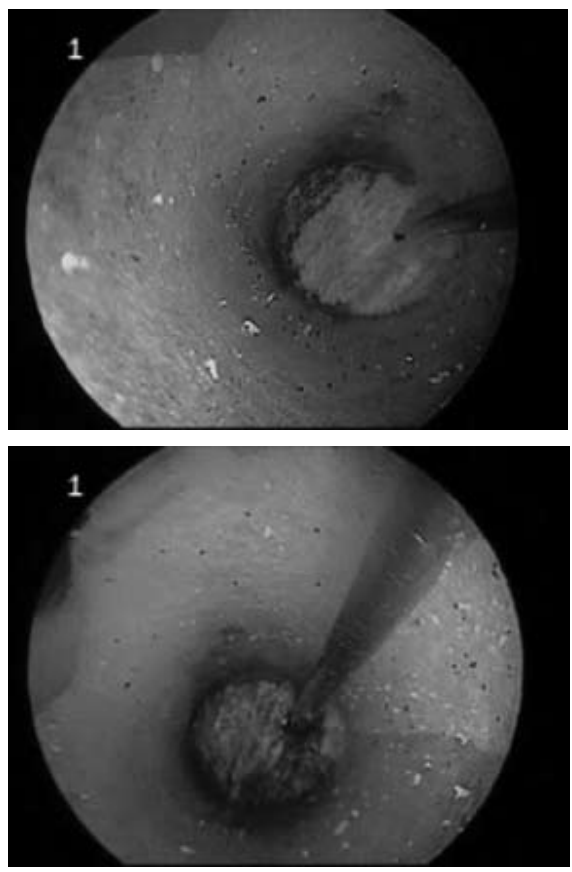

Figure 6A. model TM made out of micropore tape

Figure 7. Myringotomy done on anteroinferior

Figure 8. suction of fluid 


\section{SURGICAL INNOVATIONS AND INSTRUMENTATION}

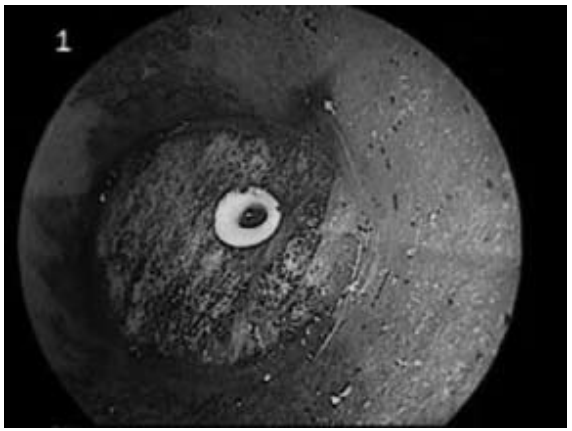

Figure 9 A. myringotomy tube in place

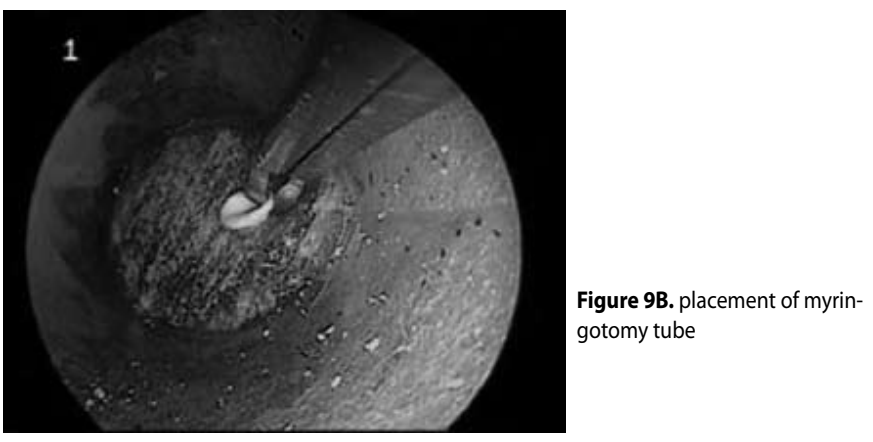

\section{DISCUSSION}

Myringotomy is a common procedure for otorhinolaryngologists. In cooperative teenagers and adults, this can be done under local anesthesia in a clinic setting. In children, general anesthesia is usually required for tympanostomy tube insertion.2 Although this is usually a straightforward procedure, difficulties during the process may be encountered. Caution must be exercised to avoid traumatizing the anterior canal wall to avoid bleeding and pain. Creation of a toolarge myringotomy might cause the tympanostomy tube to fall into the middle ear. Additional difficulty is encountered when performing myringotomy on a very small ear canal. These complexities may be avoided or minimized by the experience and skills of the surgeon. Trainees are expected to perform such procedures but the lack of materials and expensive cost of commercially available models for teaching myringotomy hamper acquisition of skills before practice on actual patients, potentially compromising patient safety.

Other models have been developed to meet the need for practicing surgical skills in myringotomy.3,4 Some use a mannequin ear to simulate the external ear canal These models either use materials that are not readily available and expensive especially in our setting. Our proposed model was basically an inexpensive system made out of readily available materials. One could easily construct his or her own myringotomy practice set by using the aforementioned materials and procedure.

The external auditory meatus or canal is composed of the lateral cartilaginous and medial osseous portions. It is approximately 2.5 $\mathrm{cm}$ from the conchal cartilage to the tympanic membrane.5 The tympanic membrane is approximately $8 \mathrm{~mm}$ wide, 9 to $10 \mathrm{~mm}$ high and $0.1 \mathrm{~mm}$ thick. Its inferior pole is oriented more medially than its superior pole by approximately 40 degrees. 5 The model described in this paper tried to emulate these dimensions of the external ear by cutting the $3 \mathrm{cc}$ syringe to $2.5 \mathrm{~cm}$ to approximate the length of the external auditory canal and beveling one end to mimic the tympanic membrane orientation. The 3-cc syringe had a diameter of $9 \mathrm{~mm}$ and approximated the dimensions of the tympanic membrane of an adult. The materials used at the beveled end could be interchanged to experience different textures for one practice on. In our experience, Micropore $^{\mathrm{TM}}$ tape seemed to best mimic the thickness and resistance of the tympanic membrane when punctured. Markers could be placed on the micropore ${ }^{\mathrm{TM}}$ prior to the myringotomy exercise to indicate the proper orientation of the tympanic membrane and the location of the malleus. After the procedure, trainees could check the accuracy of the incision based on the markers. Fluid could be placed in the depression in the modeling clay to practice suctioning fluids from the middle ear. The color and viscosity could be modified using dyes and thickeners to mimic various fluids in middle ear diseases. Gentian violet could also be used to practice and simulate administration of local anesthesia. Avoidance of the ear canal wall could be practiced by placing dye on the myringotomy knife and making sure the dye was not transferred to the walls. Smaller tubes could be used to simulate pediatric ears.

Our device was very economical as most materials were readily available (or could be purchased at minimal cost) compared with commercially available mannequins and models (Table 1) with prices ranging from USD 65.006 to 950.00 .7

\section{REFERENCES}

1. Aguila KP. Self-retaining harpoon tympanostomy tube with applicator. Philipp J Otolaryngol Head Neck Surg. 2007;22(1,2): 27-30.

2 Hirsch, B. Myringoplasty and Tympanoplsty In: Myers, E, editor. Operative Otolaryngology Head and Neck Surgery. Volume II. Philadelphia: WB Saunders Company, 1997. p.1236-1245

3 Owa AO and Farell RW. Simple model for teaching myringotomy and aural ventilation tube insertion. J Laryngol Otol. $1998 \mathrm{Jul} 112(7)$ : 642-3

4 Pichechero M, Poole M., Auran M, Pichichero FA. Teaching mannequin for developing tympanocentesis skills. Outcomes management educational workshops, Inc. Available from http://www.omew.com/research/mannequin.htm

5 Ducket L. Anatomy of the Skul Base, Temporal Bone, External Ear, and Middle Ear, In: Cummings, CW, Fredrickson JM, Harker LA, Krause CJ, Schuller DE, Richardson MA, editors. Otolaryngology Head and Neck Surgery, 3rd edition. Volume IV. St Louis: Mosby; 1998. p. 2533-2546

6 The Health Care Net [homepage on the internet]. Life-size ear models [cited 2008 June 3]. Available from: http://www.thehealthcarenet.com/Models_ear_eye_sinus_ teeth.asp\#GPI225

7 Buyamag, Inc. Magnetic Therapy, Acupuncture Health Products and Supplies[homepage on the internet] . Ear Models [cited 2008 June 3]. Available from: http://www.buyamag.com/index.htm 